\title{
'Wanting' and 'Liking' Brain Mechanisms in Coaching: A qEEG Study Using the CARE Coaching Model
}

\author{
Lyra Puspa ${ }^{1,2^{*}}$, Nurhadi Ibrahim ${ }^{3}$, and Paul T. Brown ${ }^{4}$ \\ ${ }^{1}$ Salomon Centre for Applied Psychology, Faculty of Social and Applied Sciences, Canterbury Christ Church University, \\ Kent, UK; and Vanaya NeuroLab Brain \& Behavior Research Center, Jakarta, Indonesia \\ ${ }^{2}$ Vanaya NeuroLab Brain \& Behavior Research Center, Jakarta, Indonesia \\ ${ }^{3}$ Department of Medical Physiology, Faculty of Medicine; Medical Technology Cluster IMERI; and Neuroscience \& Brain \\ Development Cluster IMERI; Universitas Indonesia, Jakarta, Indonesia \\ ${ }^{4}$ Organizational Neuroscience, Monarch Business School Switzerland.
}

\section{A R T I C L E I N F O}

\section{Article history:}

Received 19 August 2019

Received in revised form 9

October 2019

Accepted 16 October 2019

Available online 31 October 2019

\section{Keywords:}

Coaching,

Wanting and liking,

qEEG,

Delta,

Beta-gamma.

\section{*) Corresponding author:}

lyra@vanaya.co.id

\begin{abstract}
A B S T RA C T
Introduction: Coaching has become increasingly popular for leadership development and behavioral change in organizations. Recent studies suggest that motivation is essential in enhancing the effectiveness of workplace coaching. A number of studies revealed that delta and beta-gamma oscillations are associated with the human motivational process through 'wanting' and 'liking' mechanisms. However, the brain mechanisms of motivation in coaching have not been studied. This preliminary study is the first attempt to explore the 'wanting' and 'liking' mechanism of coaching, by investigating the activity of the delta and beta oscillations during a face-to-face coaching session through quantitative electroencephalogram (qEEG).

Methods: Six male, right-handed, middle managers of an organization (mean age $=31.6$ ) were recruited voluntarily as participants. A multichannel EEG (19 electrodes, 10/20 System) was used to record brain activity in both the resting state and the continuous 45-minute coaching session whilst using the CARE Model. The artifact-free EEG data were then quantified using wavelet analysis to obtain induced band power.

Results: Significant increase was shown in delta and beta-gamma activities throughout the coaching session. Increased delta absolute power was found in the frontal, parietal, and occipital regions, whilst increased beta-gamma activity was significantly detected in the frontal, posterior temporal, and occipital regions.

Conclusion: This preliminary result suggests that coaching, with regard to the CARE Model, induces both 'wanting' and 'liking' mechanisms simultaneously. Thus, the present findings provide the first preliminary neuroscientific underpinnings of the role of motivation in enhancing the effectiveness of workplace coaching through induced 'wanting' and 'liking' mental processes.
\end{abstract}

\section{Introduction}

Coaching has grown exponentially over the past decade as a popular intervention to develop leadership capacity within organizations. 'Coaching' is derived from the verb 'to coach' which has the root meaning of transporting valued persons from their current place to their expected destination. ${ }^{1}$ Most of the current definitions of coaching describe coaching as a means of obtaining optimum performance and changing behavior in a desirable way through a collaborative verbal conversation in order to achieve individual or organizational desired goals. ${ }^{2-4}$ In short, the essence of coaching in a leadership development context is about facilitating self-directed change and growth, either for short-term performance improvement or sustained behavioral change in the long run.

As a means of facilitating individual change to achieve the desired individual or organizational goals, enhancing motivation for change is essential in any workplace coaching. Human motivation is critically important for coaches to understand since it impacts significantly upon coachee's readiness to change. Extensive studies have provided evidence for the impact of coaching in motivational aspects, such as hope $;, 6$ self-concordance and commitment; ${ }^{7}$ desires, passions, aspirations, and purposes $;{ }^{4}$ self- 
efficacy $;{ }^{8}$ as well as resilience and goal-attainment. ${ }^{9}$ Whilst a wide-array of the motivational impacts of coaching have been studied, the coachee's neurobiological processes of increased motivation during coaching conversation are still unclear.

Evidence from neuroscience studies suggested that human behavior to pursue a desired outcome is associated with separate neural networks involving both the motivation to attain a rewarding outcome ('wanting') and the hedonic feeling of sensory pleasure ('liking') upon the experience of the rewarding outcome. A recent systematic review considered 'wanting' and 'liking' as mental (neurological) processes, as well as utility. ${ }^{10-13}$

As mental processes, both 'wanting' and 'liking' are described as pre-conscious processes. However, 'liking' is considered as an affective process, whilst 'wanting' may involve cognitive desire that is stimulated by explicit thoughts of the expected outcome. As neurological process, 'wanting' is the result of activity in the mesolimbic dopamine system, whereas 'liking' relates to more limited, opioid hedonic hotspots involving activity within the nucleus accumbens (NAcc) and the ventral pallidum. When considered as utility, it was suggested that 'wanting' can be regarded as a decision utility (the degree of decision concerning which types of outcome to be chosen) and 'liking' is associated with experienced utility (the hedonic pleasure of actual reward consumption). Both types of utility are considered to be independent of predicted utility (the expectation of hedonic pleasure toward future reward).

Ample evidence in EEG studies revealed that waking delta (1-4 Hz) and beta-gamma $(20-35 \mathrm{~Hz})$ oscillations are associated with the motivational process. Such studies have found that enhanced delta-band activity accompanied dopamine release in the mesolimbic dopamine pathway during reward deficiency and craving. ${ }^{14,15}$ Meanwhile, a number of recent studies in human reward processing showed an increase of beta-gamma activity upon experiencing actual positive reward outcomes. It is argued that beta-gamma oscillatory activity mediates the interplay of attentional system, memory, and reward processing. ${ }^{16}$ Such EEG evidence suggests that 'wanting' is associated with enhanced activity in delta and 'liking' is correlated with increased beta-gamma activity.

In spite of numerous studies in animal models that have suggested a separation between 'wanting' and 'liking' mechanisms, Pool and colleagues have proposed that 'wanting' and 'liking' are not separated in human reward processing due to the existence of expected pleasantness that underlies cognitive desires. ${ }^{10,11}$ Considering the vital role of motivation in coaching to change behavior and improve performance in humans towards the desired outcomes, we argue a priori that 'wanting' and 'liking' brain mechanisms are activated simultaneously in coaching. However, despite the extensive evidence of descriptive motivational studies both in coaching psychology and the affective neuroscience literature, extensive searching indicated that no empirical studies were available regarding the neural correlates of human motivation within a coaching conversation. Inspired by this gap of evidence, we decided to investigate whether a brain oscillatory signature of 'wanting' and 'liking' is induced during a face-toface workplace coaching conversation.

This study is the first attempt to explore the waking delta and beta-gamma oscillations during an actual coaching conversation. It uses the CARE Model as a way of systematizing the coaching process. The CARE model consists of four sequential stages of Clarity, Awakening, Resolution, and Empowerment. ${ }^{17}$ These discrete sequential stages within the CARE Model enabled this research to analyze which type of specific conversation within a continuous coaching session it is that may elicit the brain mechanisms of motivation. Using quantitative electroencephalography (qEEG) to analyze the data, we hypothesized that significant delta and beta activities would be induced in at least one of the stages of the CARE Model during the coaching session. To the extent that 'wanting' delta and 'liking' beta-gamma cortical networks have not been much explored during any social interaction such as coaching, we also expected to discover which cortical regions are activated during motivational 'wanting' and 'liking' process within the overall coaching conversation and at what particular stage in a coaching session.

\section{Methods \\ Participants}

Six healthy males who were considered as high potential leaders within a commercial organization participated voluntarily in the study. Their age was within the range of 30-34 years with a mean age in 31.6 years. All of them were right-handed and held middle management positions in their organization. Informed consent was given and signed by the participants before the experiment began which had been approved ethically by the Professional Development Foundation (PDF) on behalf of Canterbury Christ Church University (CCCU), United Kingdom, and the Medical Research Ethics Committee in the Faculty of Medicine of Universitas Indonesia, Indonesia

\section{Experimental Design}

The study used a within-subject design to minimize the risk of individual differences and variance within groups at this preliminary stage of enquiry. The EEG data were recorded in two stages: during a resting condition and during the coaching session. To examine the significant impact of a coaching intervention in eliciting 'wanting' and 'liking' mechanisms, the coaching state of all participants as the intervention group was compared to their resting state. In such a way all experimental subjects were considered as their own control.

Stimulus was given through a simultaneous twoways coaching conversation which referred to the CARE Model. ${ }^{17,18}$ Consisting of four stages - Clarity, Awakening, Resolution, and Empowerment (Figure 
1) - each of the stages involved specific questions which were each considered as a stimulus that could cause action potentials in the brain. The Clarity stage began with the question of 'What do you want?' which facilitated the goal-setting process. The second stage, Awakening, asks 'Why is it important?' and is intended to trigger a reflective process and ignite a new awareness that comes from the question. This stage was followed with the Resolution stage asking
'How will you achieve it?' which provides space within the coaching conversation for ideation of a workable action plan. Lastly, the coaching session was closed with the Empowerment stage that started with the question of 'When will you finish it?', was designed to stimulate the coachee's accountability and commitment.

\section{EEG Recording}

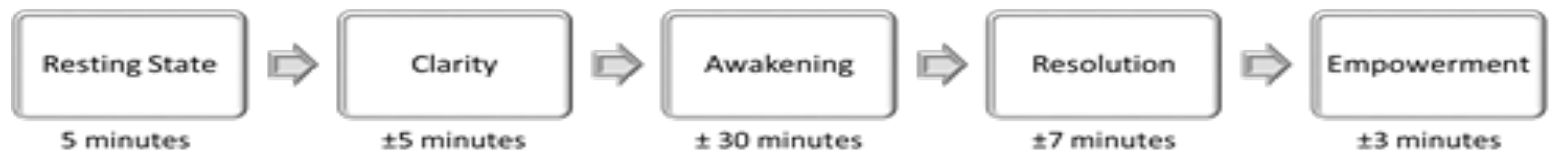

Figure 1. Experimental Design using the CARE Model

Nineteen EEG channels of the International 1020 system were used in this study (Deymed TruScan 32 Acquisition EEG System) using the sampling rate of $128 \mathrm{~Hz}$. A clinical EEG cap with soft silicone electrode cups (ANT Neuro waveguardTM connect) was used to ensure a reliable EEG recording during speech and motion as well as a comfortable EEG recording condition during coaching. The EEG assessment was started with 5-minutes resting state and continued with an approximately 45-minutes coaching session. All electrode impedances were kept below $10 \mathrm{k} \Omega$ and notch filter was applied to 50 $\mathrm{Hz}$ for the initial noise filtering.

\section{Data Processing and Analysis}

Data were pre-processed using EEGLAB Toolbox. ${ }^{19}$ EEG data were resampled to $250 \mathrm{~Hz}$, filtered with band pass filter to $1-45 \mathrm{~Hz}$ and epoched to 3 seconds. Artefacts were removed through multiple stages of digital pre-processing which consists of removing bad channels, channel interpolation, line noise removal, automatic epoch rejection, and automatic independent component analysis (ICA).

After the pre-processing phase, EEG data were processed further in Brainstorm. ${ }^{20}$ Band power was calculated for the delta $(1-4 \mathrm{~Hz})$ and betagamma $(20-35 \mathrm{~Hz})$ frequency bands that relate to the EEG frequency of 'wanting' and 'liking'. 14,16 The resulting power values of both the resting state and the coaching state were analyzed through Morlet Wavelet analysis in order to obtain a reliable spectro-temporal representation of induced absolute band power. ${ }^{21,22}$ Finally, statistical analysis over the wavelet transformed EEG data was done using the Wilcoxon signed rank permutation t-test by comparing the coaching state condition of each CARE stage with the resting state condition in order to produce t-test brain topography for further regionwise analyses and data interpretation.

\section{Results}

\section{Delta Oscillatory Activities}

Workplace coaching in a face-to-face setting seems to activate delta oscillations over a wide spread of cortical regions (Figure 2). When the absolute power of delta (1-4 Hz) frequency during the active coaching state was compared to the absolute delta power of the resting state using the Wilcoxon signedrank permutation t-test, then based on the t-test brain topography result, the delta activity during coaching was increased significantly $(p<0.05)$ in the left prefrontal, bilateral frontal (F7, F4, and F8), central parietal $(\mathrm{Pz})$, and bilateral occipital (O1and $\mathrm{O} 2)$ regions (Figure $2 \mathrm{a}$ ).

Further analyses to explore the delta oscillation activity in each CARE stage revealed that enhanced delta was elicited throughout all CARE stages (Figure 2b). Most of the significant difference in delta frequency occurred in bilateral frontal regions (F7 and F8), though Empowerment stage that did not show significant power difference at F8. Delta activities in the Clarity stage were detected in some other cortical areas, including left prefrontal (Fp1) and central parietal $(\mathrm{Pz})$ regions. Increased deltaband power in the Empowerment stage was also found in the left occipital region $(\mathrm{O} 1)$.

\section{Beta-Gamma Oscillatory Activities}

Increased beta-gamma $(20-35 \mathrm{~Hz})$ frequency absolute power was elicited during the coaching conversation compared to the resting state condition (Figure $3 \mathrm{a}$ ). The significant increase $(p<0.05)$ of beta-gamma activity was found in the bilateral prefrontal (Fp1 and Fp2), bilateral frontal (F7, F4, and F8), bilateral posterior temporal (T5 and T6), and bilateral occipital (O1 and $\mathrm{O} 2)$ regions

When the beta-gamma significant power difference t-test was applied to each CARE stage, it showed that the activation of frontal, posteriortemporal, and bilateral occipital regions occurred consistently across all stages of the CARE Model (Figure 3b). In all coaching stages, induced betagamma band power is found consistently in F8, T5, T6, O1, and O2. Concurrently there were some variations whilst using electrode-wise specific observation. Pre-frontal beta-gamma activity was found in the right hemisphere (Fp2) during the Awakening, Resolution, and Empowerment stages, whereas coaching conversation in the Clarity and the Empowerment stages stimulated the left pre-frontal (Fp1) beta-gamma oscillations. 
The Clarity and Awakening stages shared a similar pattern of beta-gamma activity at F6. Meanwhile, parietal activation at P6 was only shown in the Awakening stage and temporal activation at T4 was only detected in the Resolution stage. It is interesting to notice that increased beta-gamma was most widespread across cortical regions in the Awakening stage, even though the most significant power difference was revealed in the Resolution stage.

a)
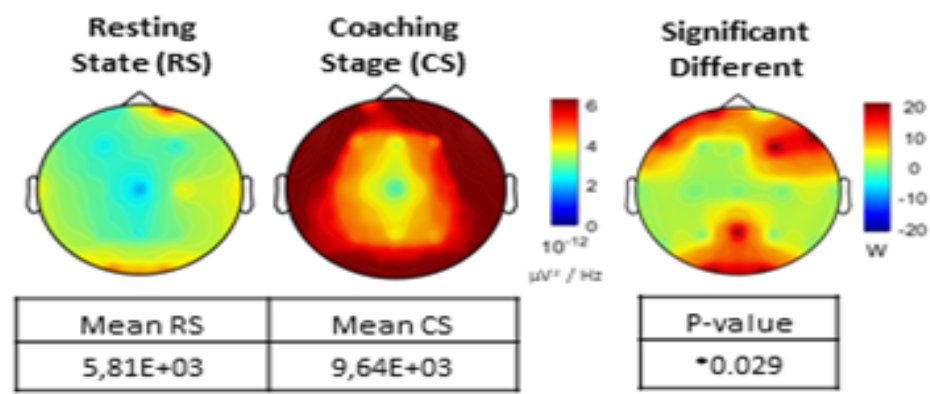

b)

\begin{tabular}{|c|c|}
\hline Mean RS & Mean CS \\
\hline $5,81 \mathrm{E}+03$ & $9,64 \mathrm{E}+03$ \\
\hline
\end{tabular}

$\cdot 0.029$

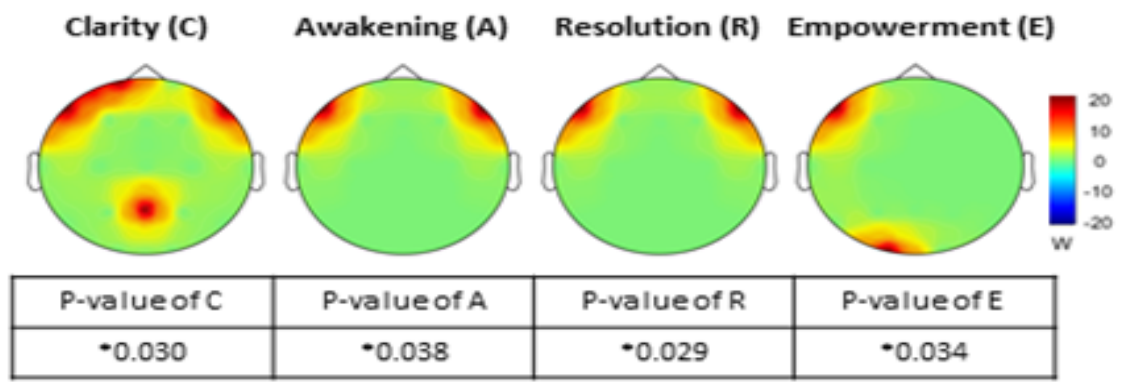

*Wilcoxon signed rankpermutation t-test $p<0.05$

Figure 2. a) Significant difference of absolute power intensity in delta oscillations between the coaching state (across all CARE stages) and the resting state; and b) Significant delta activation in each CARE stage

a)
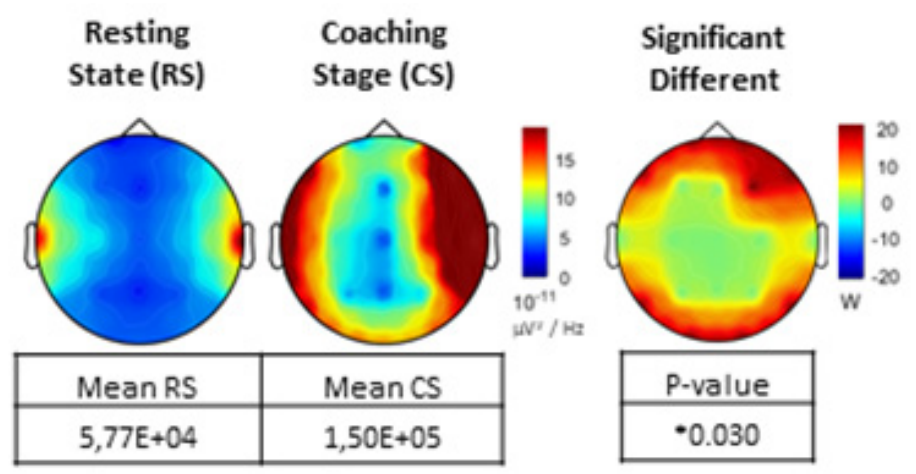

b)

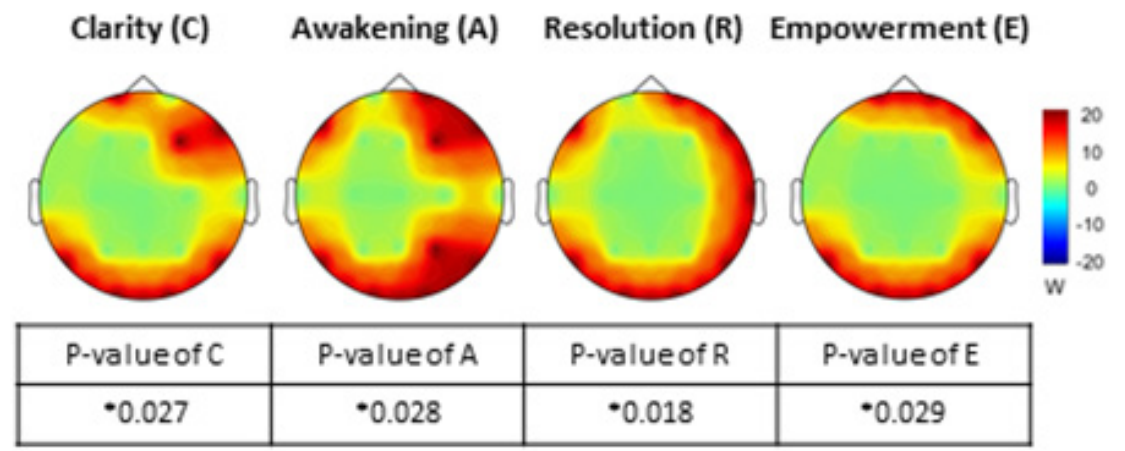

*Wilcoxon signed rank permutation t-test $p<0.05$

Figure 3. a) Significant difference of absolute power intensity in beta-gamma oscillations between the coaching state (across all CARE stages) and the resting state; and b) Significant beta-gamma activation in each CARE stage 


\section{Discussion}

This study showed that both delta (1-4 Hz) and beta-gamma (20-35 Hz) EEG activity was activated significantly during coaching conversation in comparison with the resting state. Referring to the earlier studies investigating animal affective process on 'wanting' delta and 'liking' betagamma, this preliminary result indicates that a coaching intervention using the CARE Model stimulates both 'wanting' and 'liking' mechanisms simultaneously. ${ }^{14-16}$ This finding supports the idea that 'wanting' and 'liking' do not work separately in human reward processing, particularly in a social interaction context such as when experiencing a coaching conversation. ${ }^{10}$

It is interesting to notice that coaching seemed to ignite the simultaneous activation of delta and beta-gamma starting from the Clarity stage of the CARE Model. Since the essence of the Clarity stage within the CARE Model lies in facilitating the coachee to create a clear conceptual outcome for both the long-term goal and the immediate objective of the particular session, we propose the idea that the 'wanting' delta-band activity was induced by the goalsetting process during coaching. One the coach facilitated the coachee to set a clear outcome, the coachee desired to achieve the expected goal. This desire may be driven by two conditions, either the awareness of the gap between current condition and the future expected outcome or the hedonic liking experience when the coachee imagined the expected goal. It is still unclear whether one of the conditions is more dominant over another in a coaching relationship.

The simultaneous interaction between 'wanting' and 'liking' in this study also indicates that coaching may induce goal-directed 'wanting' and the expected pleasantness that underlies cognitive desires. This indication is derived from three arguments.

First, coaching is a goal-focused activity where the coach facilitates the coachee to establish an expected outcome and create a workable plan to achieve the desired goal. ${ }^{2,3}$ The conscious goal orientation of a coaching conversation suggests that waking delta-band activity during the coaching conversation seemed to be more inclined to goal-directed explicit 'wanting' in human motivational process instead of cue-triggered Pavlovian incentive salience or implicit wanting concept seen in animal investigations. ${ }^{10}$

Second, the decision utility of explicit 'wanting' in coaching was not independent of both predictive or experienced utility of 'liking'. Most animal studies have defined 'liking' as the hedonic state upon the actual experience of a positive reward. It is interesting to notice that beta-gamma activity that is associated with 'liking' was found significantly during coaching conversation where the expected future state that was decided by the coachee was not necessarily experienced in the past. Considering the elicitation of significant activity in the parieto-occipital region which is associated with visual perception, this study implied that coaching questions may ignite the hedonic pleasure of experiencing the visuospatial representation of coachee's desired future state - 'seeing' a desired future. Thus, the beta-gamma activity during coaching seemed to be more associated with the predictive utility of 'liking' that is driven by imagination than experienced utility that is driven by past hedonic experience. We also argue that the expected pleasantness of imagined future reward is a major compound of predictive utility in any coaching conversation. Hence, it is proposed that coaching may stimulate the cognitive desires through both explicit 'wanting' and predictive utility that underlies self-driven motivation in humans. Figure 4. illustrates this proposed idea schematically.

Thirdly, the activation of delta-band in the frontal region throughout the coaching sessions and right across the CARE stages may signify that the 'wanting' mechanism is associated with delta activities in the mesolimbic dopamine system involving NAcc, amygdala, and medial frontal cortex. Meanwhile, the enhanced delta in central parietal and occipital regions is similar to the delta activity pattern during orgasm and sexual arousal induced by imagery. ${ }^{14}$ Since coaching often involves a visioning process of expected goals and future possibilities, it implies that coaching may trigger arousal that drives the eagerness to acquire the imagined future state as the expected reward. On the other hand, the ample evidence from animal studies that 'liking' hedonic hotspots involving the NAcc, ventral pallidum, and parabrachial nuclei in the brainstem opens the possibility that in humans such arousal might be associated with the frontal-posterior activation of beta-gamma frequency as seen in this study. Since this is the first attempt to explore the 'wanting' delta and 'liking' beta-gamma mechanisms in coaching, such speculation will need to be investigated in future empirical studies. However, this study may open the possibilities of waking delta and betagamma oscillations in frontal and occipital regions being explored further as the EEG correlates or brain signature of cognitive desire in the motivational process during coaching conversations.

Previous studies have revealed that the increase in high beta - low gamma oscillatory activity represents a brain signature of 'liking' toward positive outcomes. ${ }^{16}$ The more unexpected the positive reward, the greater the beta-gamma power activity. In this study, the beta-gamma increased activity was found throughout all coaching stages within the CARE Model; and the widest spread of its cortical activity was shown in the Awakening stage. A recent report regarding gamma-band activity during the Awakening stage of the CARE Model argued that coaching may induce mindful reflective thinking processes that lead to self-awareness. ${ }^{18}$ The deep-reflective CARE process that elaborates coaching exploration around 'Why is it important?' seemed to stimulate the hedonic pleasure of the unexpected rewarding 'aha' moment. The 'aha' moment of awareness is one of the essential outcomes of workplace coaching that creates positive experience. ${ }^{2,23}$ This study then offers the possibility that the significant 'liking' beta-gamma responses that occurred in the Awakening stage of the CARE Model represent unexpected positive reward as evidenced in the 'aha' moment of new awareness. 


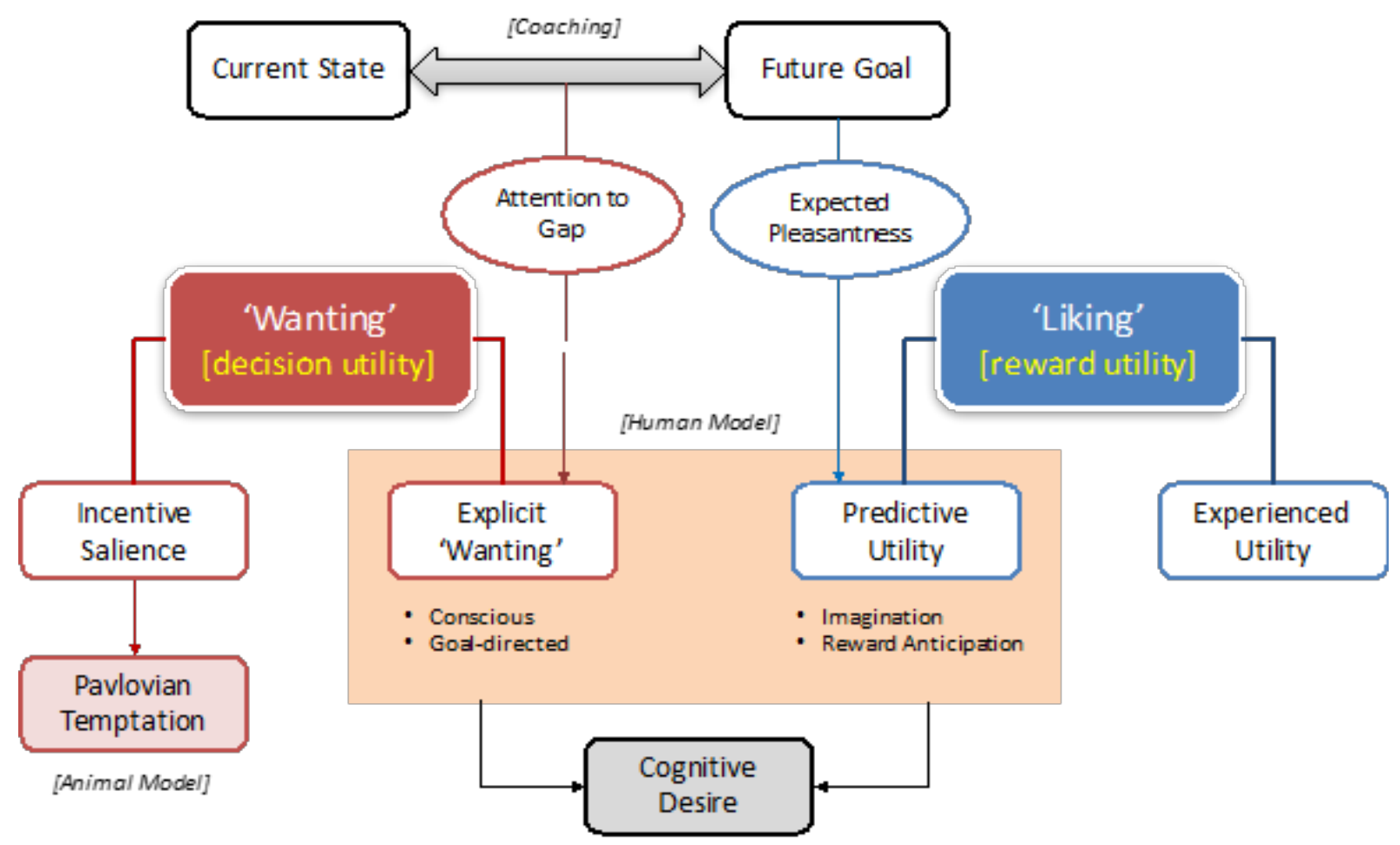

Figure 4. Illustration of the proposed simultaneous 'wanting' and 'liking' mechanisms in motivational process during coaching conversation

\section{Conclusion}

This study indicates that 'wanting' and 'liking' may work simultaneously in coaching intervention regarding the CARE Model through the stimulation of goal-directed cognitive desire. Some possibilities of there being a motivational brain signature of coaching were proposed. Thus, the present findings provide the preliminary neuroscientific underpinning of the role of motivation in enhancing the effectiveness of workplace coaching through induced 'wanting' and 'liking' mental processes.

However, further study is required to examine the specific subcortical structures of 'wanting' and 'liking' that are stimulated by coaching. Future research with a larger number of participants is also needed to explore whether there are certain 'wanting' and 'liking' brain signature that can be generalized across various coaching models in both personal and organizational contexts. Finally, it will be a breakthrough to have an empirical exploration on how different intensity of 'wanting' and 'liking' mechanism in coaching may contribute to actual outcomes as seen in sustainable behavioral change.

\section{Conflict of Interest}

The author stated there is no conflict of interest

\section{References}

1. Witherspoon R, White RP. Executive Coaching: A Continuum of Roles. Consult Psychol J Pract Res. 1996;48(2):124-33.

2. Whitmore J. Coaching for Performance - Growing Human Potential and Purpose (4th Edition). Nicholas Brealey Publishing. 2009.

3. Grant AM. It takes time: A stages of change perspective on the adoption of workplace coaching skills. J Chang Manag. 2010;10(1):61-77.

4. Smith ML, Van Oosten EB, Boyatzis RE. Coaching for sustained desired change. In: Research in Organizational Change and Development. 2016. p. 145-73.
5. Green LS, Grant AM, Rynsaardt J. Evidence-based life coaching for senior high school students: Building hardiness and hope. Int Coach Psychol Rev. 2007;2(1):24-32.

6. Rolo CMR da C, Gould D. An intervention for fostering hope, athletic and academic performance in university student-athletes. Int Psychol Rev. 2007;2(1):44-61.

7. Burke D, Linley AP. Enhancing goal self-concordance through coaching. Int Coach Psychol Rev. 2007;2(1):62-9.

8. Evers WJG, Brouwers A, Tomic W. A quasi-experimental study on management coaching effectiveness. Consult Psychol J Pract Res. 2006;58(3):174-82.

9. Grant AM, Curtayne L, Burton G. Executive coaching enhances goal attainment, resilience and workplace well-being: A randomised controlled study. J Posit Psychol. 2009;4(5):396-407.

10. Pool E, Sennwald V, Delplanque S, Brosch T, Sander D. Measuring wanting and liking from animals to humans: A systematic review. Neurosci Biobehav Rev. 2016;63:124-42.

11. Berridge KC. Wanting and Liking: Observations from the Neuroscience and Psychology Laboratory. Inquiry. 2009;52(4):37898.

12. Tibboel H, De Houwer J, Van Bockstaele B. Implicit measures of "wanting" and "liking" in humans. Neurosci Biobehav Rev. 2015;57:350-64

13. Kohls, G., Chevallier, C., Troiani, V., and Schultz, R. T. Social "wanting" dysfunction in autism: Neurobiological underpinnings and treatment implications. J Neurodev Disord. 2012;4(1):1-20.

14. Knyazev GG. Motivation, emotion, and their inhibitory control mirrored in brain oscillations. Neurosci Biobehav Rev. 2007;31(3):377-95.

15. Knyazev GG. EEG delta oscillations as a correlate of basic homeostatic and motivational processes. Neurosci Biobehav Rev. 2012;36(1):677-95.

16. Marco-Pallarés J, Münte TF, Rodríguez-Fornells A. The role of highfrequency oscillatory activity in reward processing and learning. Neurosci Biobehav Rev. 2014;49:1-7.

17. Puspa L. Coaching Style Leadership: Transformational Leadership with CARE Coaching Model. First Edition. Jakarta: Vanaya Ciaching International; 2012.

18. Puspa L, Ibrahim N, Brown PT. Increase in gamma band $\mathrm{qEEG}$ activity during executive coaching: Some preliminary observations and possible implications. In: Major issues and other topics related to 
Mental Health, Neuroscience, and Cyber-psychology. 2018.

19. Delorme A, Makeig S. EEGLAB: an open source toolbox for analysis of single-trial EEG dynamics. J Neurosci Methods. 2004;134(1):912.

20. Tadel F, Baillet S, Mosher JC, Pantazis D, Leahy RM. Brainstorm: A user-friendly application for MEG/EEG analysis. Comput Intell Neurosci. 2011;1-13.

21. Bruns A. Fourier-, Hilbert- and wavelet-based signal analysis: Are they really different approaches? J Neurosci Methods. 2004;137:32132.

22. Scarpelli S, Marzano C, D’Atri A, Gorgoni M, Ferrara M, De Gennaro L. State-or trait-like individual differences in dream recall: Preliminary findings from a within-subjects study of multiple nap REM sleep awakenings. Front Psychol. 2015;6:928.

23. Brand H, Coetzee M. An Explorative Study of the Experiences of the Coach and Coachee during Executive Coaching. J Soc Sci. 2017;34(3):247-56. 